\title{
Predictive Process Monitoring in Operational Logistics: A Case Study in Aviation
}

\author{
Björn Rafn Gunnarsson ${ }^{1}$, Seppe K.L.M. vanden Broucke ${ }^{1}$, and \\ Jochen De Weerdt ${ }^{1}$ \\ Research Centre for Information Systems Engineering (LIRIS) \\ KU Leuven, Naamsestraat 69, B-3000 Leuven \\ bjornrafn.gunnarsson@kuleuven.be; seppe.vandenbroucke@kuleuven.be; \\ jochen. deweerdt@kuleuven. be
}

\begin{abstract}
The research area of process mining concerns itself with knowledge discovery from event logs, containing recorded traces of executions as stored by process aware information systems. Over the past decade, research in process mining has increasingly focused on predictive process monitoring to provide businesses with valuable information in order to identify violations, deviance and delays within a process execution, enabling them to carry out preventive measures. In this paper, we describe a practical case in which both exploratory and predictive process monitoring techniques were developed to understand and predict completion times of a luggage handling process at an airport. From a scientific perspective, our main contribution relates to combining a random forest regression model and a Long Short-Term Memory (LSTM) model into a novel stacked prediction model, in order to accurately predict completion time of cases.
\end{abstract}

Keywords: Process mining - Predictive process monitoring - Operations · Long Short-Term Memory (LSTM) · Aviation

\section{Introduction}

The past decades have seen a considerable rise in the use of process aware information systems within businesses. Today, these systems have become increasingly more intertwined with the operational process they support and often leave a trace of each activity executed by organizations, recorded and stored in the form of event logs. In alignment with the development of these information systems, the field of process mining concerns itself with knowledge discovery from such event logs, aiming to extract insights and information that can be used to understand and improve the underlying processes [1].

Historically, this information has been extracted and analyzed after a business process has been executed. However, more recently, research in process mining has increasingly shifted its focus towards developing and applying process mining techniques in the context of a running process, often with the goal to deliver a prediction or recommendation with regards to the process at hand. These 
predictive process monitoring methods are constructed by analyzing historical execution traces and are then used to continuously provide users with predictions about the future of a given ongoing process execution. Predictive monitoring can therefore provide businesses with valuable information which enables them to identify violations, deviance and delays within process execution in advance making preventive measures possible $[7,19]$.

Predictive process monitoring methods have been successfully applied to a number of different tasks, including cost prediction [25], risk prediction $[5,6]$ and activity sequence predictions [11,22]. Another application that has received increased attention in the last few years is the prediction of the completion time of a process instance $[20,21]$. Predicting the completion time of a process instance has a number of business applications, including in logistic setting, where such predictions can for instance be used to indicate how long a certain step of a process will take, on the basis of which feedback can be provided to workers and end-users.

This work is inspired by the approaches suggested in [20,21]. More specifically, in a similar manner, we suggest an approach to predict the completion time of a process instance taking as input both the flow of the activities of a running case and other features describing the case. The approach suggested here was developed and applied in the real-life setting of the largest international airport in Belgium, Brussels Airport, and stems from the first phase of an analysis of the airport's baggage system, where the $\mathrm{PM}^{2}$ process mining methodology [10] (not to be confused with the similarly named project management methodology developed and endorsed by the European Commission) was utilized with the aim of predicting the completion time of transferring bags going through the airport's baggage system. The resulting proposed solution consists of a stacked predictive modeling setup which will be described in depth.

As such, the main research contributions of this paper are as follows: Firstly, the usability of the $\mathrm{PM}^{2}$ process mining framework will be illustrated in the specific setting of airport analytics. Secondly, a novel data-aware modeling approach is proposed for predicting completion time of process instances, which combines two state-of-the-art machine learning models where one model is used to obtain baseline prediction and the other is used to sequentially update the baseline predictions as activities are executed for the process instances.

The remainder of this paper is structured as follows. Section 2 provides a discussion on the literature related to this research. Section 3 details the methodology used. In Sections 4 and 5, the use of $\mathrm{PM}^{2}$ framework in the specific setting of airport analytics will be illustrated, the section will provide an explanatory and predictive analysis of the baggage system of Brussels airport. Finally, Section 6 concludes the paper and outlines directions towards future work.

\section{Related work}

The first approaches developed to improve business processes and provide support for their execution focused on measuring and monitoring activities. These approaches were attributed to a research area called "Business Activity Monitoring". 
More recently, the field of process mining has emerged which allowed for the automatic analysis of a running process instance and the prediction of different attributes relating to such instances $[14,20]$.

Since the mid-nineties, various scholars have worked on developing techniques to tackle the problem of accurately predicting the completion time of a process instance $[2,21]$. One of the first published research articles that focuses on analyzing execution duration times is [23]. However, no detailed prediction algorithm was suggested. Rather, the problem of having cross-trained resources on performance prediction was highlighted. In [8], a non-parametric regressionbased approach was suggested that exploits all data available in an event log for predicting the cycle time of a running process instance. Van der Aalst et al. [2] suggested an approach where a finite state machine is constructed, called a transition system, which combines a discovered process model with time information learned from past instances to predict completion times of running instances. In [4] a similar transition system is utilized, though where the authors propose adding nested prediction models to the system which were constructed from event logs. The model can then be used to predict the next activity of a process and the completion time of a running process instance and was found to outperform the approach suggested by [2]. More recently, scholars have started to investigate the applicability of deep learning-based approaches for a wide range of predictive monitoring tasks, stepping away from transition systembased approaches. For instance, [24] investigated the use of LSTM networks for remaining time prediction and predicting the next event of a process and found that they outperformed prior methods for predictive process monitoring.

Whereas most transition system and neural network-based approaches described above are mainly control flow oriented (meaning that the sequence of executed activities is used as the prime information to predict what will happen next), more data-driven approaches have also been investigated. In [17], Leitner et al. proposed one of the first data-aware methods for predictive process monitoring, where the authors take advantage of additional data to predict service level agreement violations. Folino et al. $[12,13]$ extended on this research by proposing a method for clustering traces according to corresponding context features and then constructing a predictive model for each cluster. Once trained, the approach can assigns new running instances to one of the clusters and then uses the corresponding model constructed for that cluster to make a prediction. In a similar manner [21], suggested a method that uses both the control and data flow perspectives jointly by constructing a process model which is augmented by time and data information in order to enable remaining time prediction.

The research most related to the approach presented in this paper is [20], where the authors propose combining the method of using both control and data flow perspectives jointly as suggested by [21] but extend on this by considering an LSTM network for predictive process monitoring as suggested by [24]. Hence, the method improves on [21] in that it does not require constructing a transition system which can be both time and memory consuming for some real-world data sets and improves on [24] in that it can consider data attributes. 
In order to combine information on control flow and data attributes, both [21] and [20] append data features to a vector containing information regarding the control flow of a process instance. The novelty of the approach suggested here is to incorporate data attributes in predictive process monitoring by using a stack of predictive models. That is, a regression-based model is used in a first step to obtain a baseline prediction for the completion time of a process instance at the beginning of its process and then in a second step the baseline predictions are updated to take into account the control flow of the process instance as it moves through the process.

\section{Methodology}

As discussed above, process mining techniques aim at extracting insights from event data as recorded by an organization's business process information system, which in turn can be used to improve the process performance. However, comparable with the related field of data mining, applying process mining in practice is not a trivial task and oftentimes involves a great deal of data wrangling and experimentation involving different viable approaches and techniques before a satisfactory outcome is reached. Just like with data mining, this has motivated researchers to develop standard methodologies that are tailored towards supporting process mining projects. One such methodology, $\mathrm{PM}^{2}$, was specifically designed for this purpose and covers a wide range of process mining and related analysis techniques [10]. The six main stages described by $\mathrm{PM}^{2}$ are shown in Figure 1.

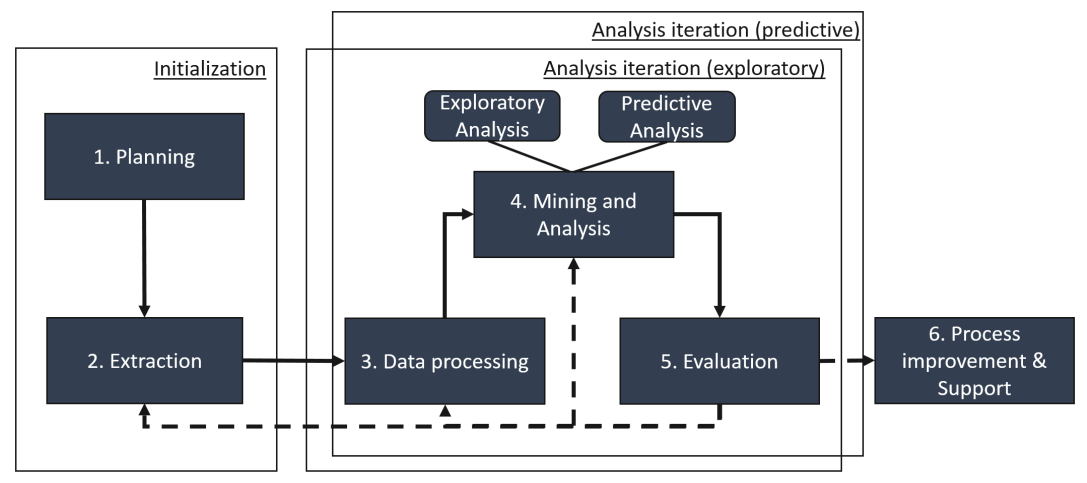

Fig. 1. Overview of the stages described by the $\mathrm{PM}^{2}$ framework

As shown in the figure the $\mathrm{PM}^{2}$ methodology can be divided into two main subgroups of stages, initialization and analysis iterations. During initialization the two first stages of methodology are carried out: planning (1), where initial research questions are defined, and (2) extraction, where the event data needed to answer the defined research questions is extracted from the business information 
systems. Next, one or more analysis iterations are performed, which focus on answering a specific research question. In general, each analysis iteration executes three stages: preprocessing the extract event data (3), analyzing the data (4), and evaluation of the results (5). If the obtained findings from the analysis iteration are deemed satisfactory, they can be used for improving the business process and supporting its operations (6).

In the following two sections the use of the $\mathrm{PM}^{2}$ methodology will be applied in a real-life setting stemming from an operational logistics context. More specifically, the methodology was used to support the application of process mining activities at Brussels Airport, the largest international airport in Belgium.

\section{Initialization}

Planning One of the many services carried out at Brussels Airport is the handling of passenger baggage. One group of bags that are of particular interest in this setting are transferring bags, which arrive at the airport with an arrival flight and are then directly rerouted to a departing flight. This group of bags can cause considerable costs if they are - for whatever reason - unable to catch their departing flight. Because of this reality, the primary goal of the research project was to obtain insights with regards to transferring bags. This was achieved by means of a two-fold analysis. First, an exploratory analysis was carried out in order to better understand the variability both in the way these bags are processed in the baggage system and the distributions of duration time. Second, a predictive model was constructed to predict the completion times of transferring bags as they enter and move throughout the luggage system, which could subsequently be used to take preventive measures in order to minimize losses caused by this group of bags.

Extraction The baggage system at Brussels Airport is almost fully automated, with bags automatically moving through different locations in the system, e.g. through a screening machine or sorting tray. A unique identifier is attached to each bag at the beginning of its journey through the system, which is scanned each time a bag arrives at a specific location in the system with the information being logged into an underlying supporting information system. This results in an event log describing complete and detailed traces for each bag, with the possibility to link this information to additional data, such as flight information, departing and originating airports, gate information for the flights, and airline information. The above information was extracted from the system for all transferring bags that were processed at Brussels Airport throughout 2018. In total, more than 1.5 million bags with their full journeys and related information were extracted from the system and form the basis of the following analysis.

\section{Analysis Iterations}

\subsection{Exploratory Analysis}

Preprocessing In order to carry out an exploratory analysis based on an event logs, three main data points need to be present. First, the unique identifier for 
each bag was used to group events to form a trace of events for each process instance (i.e. a trace describes the steps taken by one transferring bag). Second, as described above, bags are scanned every time they arrive at a location in the baggage system, leading to one event being logged in the underlying information system. To provide names of the activities in our process representation, we hence utilize the names of the locations in the system to construct a set of unique activities that bags can undergo. Third, activities are ordered within each trace by means of an exact recorded time stamp. Note that the time stamps in this setting are atomic, meaning that activities have one single time stamp and effectively have no duration. The duration for a trace as a whole is described by the time between the first time a bag is scanned and the last time, right before it exits the system. Table 1 provides an overview of descriptive statistics for the resulting processed event log.

Table 1. Descriptive statistics for the constructed event log

\begin{tabular}{lrrrr}
\multicolumn{5}{c}{ Amount } \\
Number of Traces & 1548240 & & & \\
Number of Activities & 63 & & & \\
Trace Variants & 97428 & & & \\
& Min & Max & Mean Std.dev. \\
\hline Date Range & $2018-01-01$ & $2018-12-31$ & & \\
Activities per Trace & 1 & 384 & 7.35 & 7.74 \\
Duration per Trace (minutes) & 0 & 1440 & 178.69 & 149.96
\end{tabular}

Exploratory analysis The main motivation for carrying out an explanatory analysis was to get a good understanding of the variability of how transferring bags are processed in the baggage system and the distribution of completion times of the bags. Therefore, in a first step, a variant analysis was carried out. A variant describes a group of traces that follow exactly the same end-to-end activity flow. Analyzing their distinct number and frequencies of occurrence provides good initial insights regarding the variability and main flows in the airport's baggage system. Almost 100 thousand different variants were identified in the baggage system during 2018, indicating an extreme variability in how bags are routed through the system. As shown in Figure 2, most of the process instances are actually captured by a couple of variants (e.g. the single most common variant describes about 300000 traces), followed by a "long tail" of less frequent behaviour.

In order to obtain insights into the variability of the completion times for the transferring bags the difference between the last time stamp and first time stamp in the event log for all transferring bags was computed. Figure 3 shows the distribution of the completion time of the transferring bags. As can be seen from the figure, the median completion time is around 143 minutes and two thirds of transferring bags have a completion time between 10 and 200 minutes. It can also be observed that some bags have a relatively long completion time, e.g. $5 \%$ of bags have a completion time that is longer than 400 minutes. It should be noted here that this does not necessarily indicate that these bags will miss their 


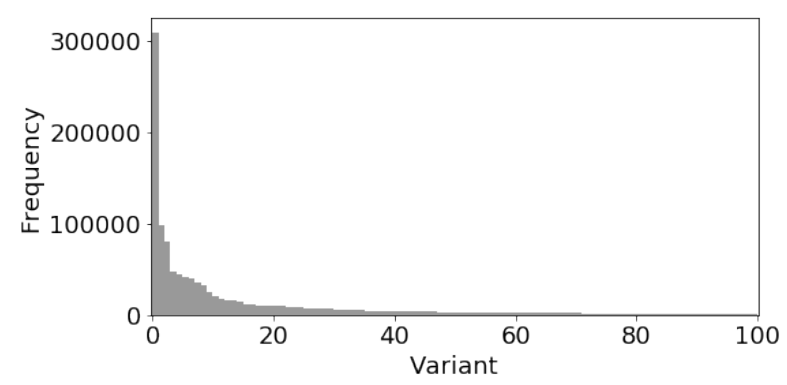

Fig. 2. Frequency overview for the 100 most frequent trace variants

departing flight, since it is not infrequent for transferring bags to be stored in the system for relatively long periods if there is a long time difference between the arrival of the flight the bag arrives on and the departure of the flight the bag departs with. The results of both the variant analysis and the analysis on completion times was validated with experts on the baggage system and luggage handling at Brussels Airport. In addition, discovery and visualization of initial process maps helped to increase understanding of the overall process as well as highlight initial bottlenecks. Also, it is important to note that this exploratory process went through a number of iterations - in line with the methodology set forward by the $\mathrm{PM}^{2}$ framework - based on feedback from domain experts and following the discovery of data quality issues stemming from the underlying information system. The event log being reported on herein is the result of a number of cleaning steps removing spurious and noisy events.

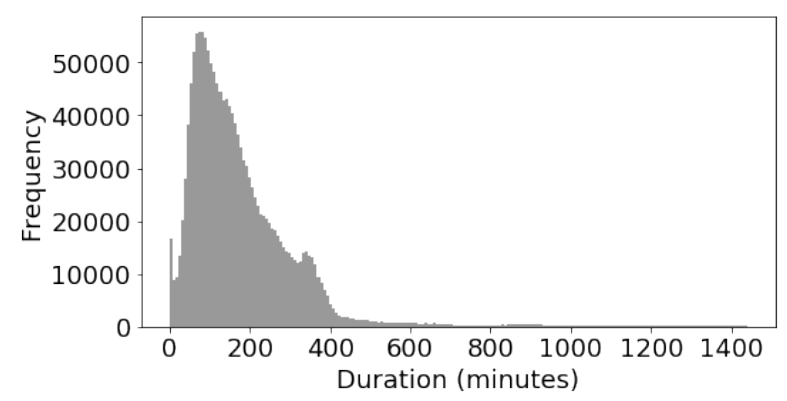

Fig. 3. Histogram of completion times for transferring bags

\subsection{Predictive Analysis}

Following the exploratory analysis, a number of predictive models were constructed in order to predict the completion times of transferring bags. First, a model was constructed only using the observed control flow for each bag, which will act as a baseline in our comparative analysis. Next, a second model was 
constructed based only on data elements known about the transferring bags when they arrive at the airport (static features). Lastly, a stacked model was constructed which combines both the static feature-based and control flow-based approaches for predicting the completion time of transferring bags.

Control Flow Model To construct the control flow-based model, a Long ShortTerm Memory (LSTM) neural network was set up to predict the completion time of traces based on last observed activities. LSTMs are a form of recurrent neural networks (RNNs), which are better suited to deal with ordered inputs compared to standard neural network architectures as they can selectively pass information across sequence steps while processing sequential data one element at a time. This enables them to model input data consisting of sequences of elements that are not independent. LSTMs are a special kind of RNNs capable of learning long-term dependencies and have demonstrated ground-breaking performance in a number of fields $[15,18]$, including process mining as previously discussed in Section 2. In order to predict the completion time of transferring bags, an LSTM network was constructed using information contained in the trace of each bag. More specifically, the complete trace of each bag was split into multiple sub-sequences of length six, as shown in Figure 4, left-padded with zeroes where necessary. A window length of six was chosen corresponding with the median number of events per trace. This collection of instances was then one-hot encoded and used to train a LSTM regression model, using a mean absolute error loss.

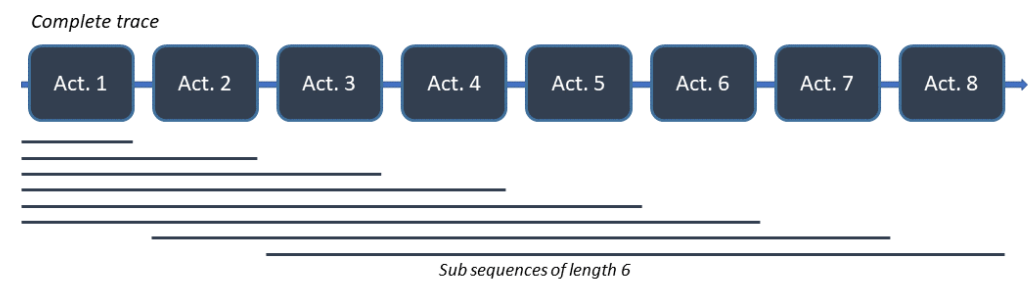

Fig. 4. An example of a complete trace and its sub-sequences

Static Features Model In a second attempt to predict the completion time of transferring bags, a random forest regressor was trained on a feature matrix consisting of data elements know about the bags when they arrive at the airport. Random forests are a well-known ensemble method originally suggested by [3] and have shown great performance in many areas when dealing with traditional, tabular data sets. In short, this method constructs a set (called the "forest") of decision trees during training and uses that to make predictions. The following features were incorporated in this model to describe bags: (i) time related information (i.e. hour of the day, day of the week, month of the year), (ii) information regarding their arrival flight (e.g. airline, previous airport), and (iii) 
similar information regarding their departing flight. Remark that no control flowbased features were considered in this model, so that the predictions provided by this model remain unchanged as a bag moves throughout the system and starts to generate events. This is a deliberate choice, as we aim to rely on the LSTM model constructed above to derive control flow-based information, rather than manually performing feature engineering over the traces, since this approach would easily lead to an explosion of features. As such, we opt here for a best of both worlds approach, where a well-understood modeling approach (random forests) is used to provide a robust baseline prediction as an instances arrives and can relatively easily explain its reasoning behind a certain prediction. In this setting for instance, it was observed that the random forest model was able to pick up on hours of the day and certain airlines as being the main important factors influencing completion times. Next, to provide more granular, precise predictions as a bag starts generating activities, the baseline prediction of the random forest has to be updated based on control flow information. To do so, we will utilize the LSTM-based model as described above, which is better suited to work with the more complex sequence data, automatically performing feature engineering as it is being trained. The two models are combined in a "stacked setup" as described below.

Stacked Combined Model The final model considered for predicting the completion times of transferring bags is constructed using a stacked approach as motivated above. Previous approaches for combining information on control flow and data attributes for predicting completion time of process instances commonly do so by appending data features to a vector containing information on the control flow of each process instance. Here, a stacked model is trained in the following manner: first, the random forest regressor is used to obtain initial baseline estimates of the completion time of transferring bags. Next, a new LSTM model was trained, now with the goal to learn how much the initial baseline predictions deviate from the actual completion times of the transferring bags (i.e. to learn the residuals). On the input side, the LSTM utilizes the same features as the baseline "Control Flow Model" setup described above, with one additional feature per instance being provided with its value set equal to the baseline prediction of the random forest. The final prediction of the stacked model is then obtained by adding the estimated deviation of the prediction random forest, estimated by the LSTM, to the initial baseline prediction of the random forest.

Implementation and Results To train the three models previously discussed in this section, $75 \%$ of transferring bags in 2018 were selected randomly, with the remaining $25 \%$ being placed in a hold-out test set to perform a final evaluation comparison. The LSTM models were constructed using two LSTM layers, each having 32 hidden units and trained using the Nadam optimizer [9]. The random forest regressor was constructed using an ensemble of 50 decision trees. Training time encompassed 24 hours for the LSTM-based models and 90 minutes for the random forest. A comparison of the obtained results for each of the three models considered here is shown in Figure 5. 


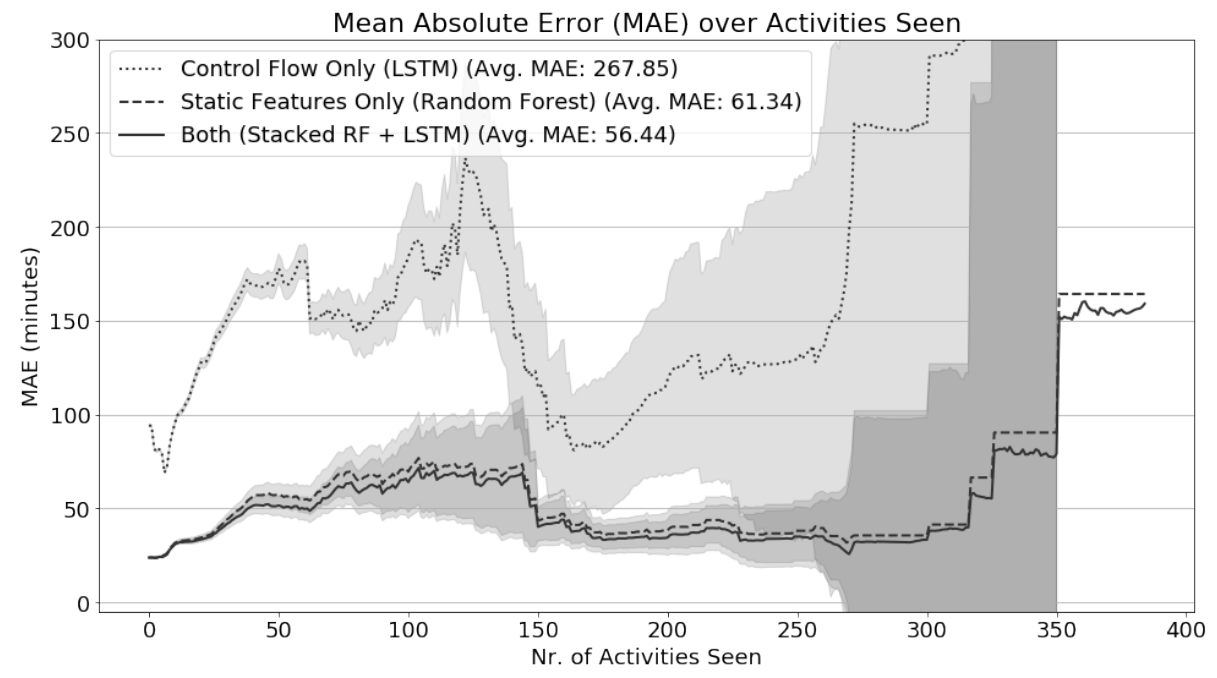

Fig. 5. Comparative overview of the three modeling approaches. The mean absolute error for each approach is shown with $95 \%$ confidence intervals over the different number of activities seen so far, together around the mean of absolute errors per different time step. The stacked model is able to improve upon the static modelling approach, especially as traces continue and become lengthier. The difference of means of the stacked versus static MAEs (56.44 versus 61.34) is significant at a $95 \%$ confidence level using a correlation-adjusted procedure to compare dependent models [16].

Various novel insights can be obtained from this comparison. First, the control flow-based LSTM network performs drastically worse than both the static featurebased random forest and the stacked model proposed here. As shown in the figure the model has an average mean absolute error (MAE) of around 268 minutes which is considerably higher than the average MAE of the two other approaches considered here. This suggests that completion times of transferring bags are heavily affected by static features such as time related information regarding when the bag arrives at the airport, information on the airline that arrived and departed with bag and the airport the bag is arriving from and departing to. Research on predictive process monitoring has increasingly focused on data-aware process monitoring where the control flow of process instances is combined with non-process related features in order to improve predictive accuracy. The stacked method suggested in this paper does so by combining a well-understood modelling approach, namely random forests, which provides a robust and explainable baseline prediction based on static information, with the deeper learning mechanism provided by an LSTM model to focus on the more complex inputs, namely control flow information. This stacked method is the best performing method of all method considered here and has a average MAE of 61 minutes which is an improvement of around 5 minutes compared to the static feature only-based approach. 


\section{Conclusion and Future Work}

For the past decade, research on predictive process monitoring techniques has increasingly focused on developing data-aware methods that combine information on processes with other non-process related information. A novel stacked dataaware predictive process monitoring method was suggested in this paper. The method stacks two predictive model where a robust baseline model, random forest regressor, is used to provide baseline prediction based on static feature information and an LSTM neural network is used to sequential update the initial baseline prediction as the process instance starts generating activities. This approach was developed and applied in a real-life setting where the model was used to predict the completion time of transferring bags at Brussels Airport. The constructed method outperforms both a static feature based approach and a control flow based approach for predicting completion time. The paper also illustrated the usability of the $\mathrm{PM}^{2}$ process mining framework in the specific setting of airport analytics. This research stems from the first phase of analysis into Brussels Airports baggage system and a number of future research avenues can be explored in future work. A key motivation for including a static feature based model in the proposed stacked setup is that its prediction can be relatively easily explained which provides valuable insights into features that influence completion times of the bags. An alternative approach entails including all static features in a single sequential model. This approach has been utilized for predictive process monitoring and therefore a direct comparison of the two methods would be of interest. Also, neural network based models include a number of hyper-parameters that can be tuned. It would therefore be of interest conduct a thorough grid search over a large tune grid of values for these parameters (including the LSTM architecture setup and length of the subsequences considered) in an attempt to further improve the performance of the LSTM based networks. Lastly, the research presented here focuses on predicting the duration times of transferring bags. However, it would also be of interest to extend on the model to predict the next likely activities in a running instance.

\section{References}

1. Van der Aalst, W., Weijters, T., Maruster, L.: Workflow mining: Discovering process models from event logs. IEEE Transactions on Knowledge and Data Engineering 16(9), 1128-1142 (2004)

2. Van der Aalst, W.M., Schonenberg, M.H., Song, M.: Time prediction based on process mining. Information systems 36(2), 450-475 (2011)

3. Breiman, L.: Random forests. Machine learning 45(1), 5-32 (2001)

4. Ceci, M., Lanotte, P.F., Fumarola, F., Cavallo, D.P., Malerba, D.: Completion time and next activity prediction of processes using sequential pattern mining. In: International Conference on Discovery Science. pp. 49-61. Springer (2014)

5. Conforti, R., De Leoni, M., La Rosa, M., Van Der Aalst, W.M.: Supporting riskinformed decisions during business process execution. In: International Conference on Advanced Information Systems Engineering. pp. 116-132. Springer (2013)

6. Conforti, R., de Leoni, M., La Rosa, M., van der Aalst, W.M., ter Hofstede, A.H.: A recommendation system for predicting risks across multiple business process instances. Decision Support Systems 69, 1-19 (2015) 
7. Di Francescomarino, C., Ghidini, C., Maggi, F.M., Milani, F.: Predictive process monitoring methods: Which one suits me best? In: International Conference on Business Process Management. pp. 462-479. Springer (2018)

8. van Dongen, B.F., Crooy, R.A., van der Aalst, W.M.: Cycle time prediction: when will this case finally be finished? In: OTM Confederated International Conferences" On the Move to Meaningful Internet Systems". pp. 319-336. Springer (2008)

9. Dozat, T.: Incorporating nesterov momentum into adam. Tech. rep., Stanford University (2016), http://cs229.stanford.edu/proj2015/054_report.pdf

10. van Eck, M.L., Lu, X., Leemans, S.J., van der Aalst, W.M.: PM²: A process mining project methodology. In: International Conference on Advanced Information Systems Engineering. pp. 297-313. Springer (2015)

11. Evermann, J., Rehse, J.R., Fettke, P.: Predicting process behaviour using deep learning. Decision Support Systems 100, 129-140 (2017)

12. Folino, F., Guarascio, M., Pontieri, L.: Discovering context-aware models for predicting business process performances. In: OTM Confederated International Conferences" On the Move to Meaningful Internet Systems". pp. 287-304. Springer (2012)

13. Folino, F., Guarascio, M., Pontieri, L.: Discovering high-level performance models for ticket resolution processes. In: OTM Confederated International Conferences" On the Move to Meaningful Internet Systems". pp. 275-282. Springer (2013)

14. Golfarelli, M., Rizzi, S., Cella, I.: Beyond data warehousing: what's next in business intelligence? In: Proceedings of the 7th ACM international workshop on Data warehousing and OLAP. pp. 1-6. ACM (2004)

15. Hochreiter, S., Schmidhuber, J.: Long short-term memory. Neural computation 9(8), 1735-1780 (1997)

16. Jolliffe, I.T.: Uncertainty and inference for verification measures. Weather and Forecasting 22(3), 637-650 (2007)

17. Leitner, P., Wetzstein, B., Rosenberg, F., Michlmayr, A., Dustdar, S., Leymann, F.: Runtime prediction of service level agreement violations for composite services. In: Service-oriented computing. ICSOC/ServiceWave 2009 workshops. pp. 176-186. Springer (2009)

18. Lipton, Z.C., Berkowitz, J., Elkan, C.: A critical review of recurrent neural networks for sequence learning. arXiv preprint arXiv:1506.00019 (2015)

19. Márquez-Chamorro, A.E., Resinas, M., Ruiz-Cortés, A., Toro, M.: Run-time prediction of business process indicators using evolutionary decision rules. Expert Systems with Applications 87, 1-14 (2017)

20. Navarin, N., Vincenzi, B., Polato, M., Sperduti, A.: Lstm networks for data-aware remaining time prediction of business process instances. In: 2017 IEEE Symposium Series on Computational Intelligence (SSCI). pp. 1-7. IEEE (2017)

21. Polato, M., Sperduti, A., Burattin, A., de Leoni, M.: Data-aware remaining time prediction of business process instances. In: 2014 International Joint Conference on Neural Networks (IJCNN). pp. 816-823. IEEE (2014)

22. Polato, M., Sperduti, A., Burattin, A., de Leoni, M.: Time and activity sequence prediction of business process instances. Computing 100(9), 1005-1031 (2018)

23. Reijers, H.A.: Case prediction in bpm systems: a research challenge. Journal of Korean Institute of Industrial Engineers 33(1), 1-10 (2007)

24. Tax, N., Verenich, I., La Rosa, M., Dumas, M.: Predictive business process monitoring with lstm neural networks. In: International Conference on Advanced Information Systems Engineering. pp. 477-492. Springer (2017)

25. Tu, T.B.H., Song, M.: Analysis and prediction cost of manufacturing process based on process mining. In: 2016 International Conference on Industrial Engineering, Management Science and Application (ICIMSA). pp. 1-5. IEEE (2016) 